\title{
Non-Gaussian statistics of soliton timing jitter induced by amplifier noise
}

\author{
Keang-Po Ho \\ Graduate Institute of Communication Engineering, National Taiwan University, Taipei 106, Taiwan
}

Received April 29, 2003

\begin{abstract}
Based on first-order perturbation theory of the soliton, the Gordon-Haus timing jitter induced by amplifier noise is found to be non-Gaussian distributed. Both frequency and timing jitter have larger tail probabilities than Gaussian distribution given by the linearized perturbation theory. The timing jitter has a larger discrepancy from Gaussian distribution than does the frequency jitter. (C) 2003 Optical Society of America OCIS codes: $060.5530,190.5530,060.4370$.
\end{abstract}

The Gordon-Haus (GH) timing jitter of the fiber soliton that is due to amplifier noise is usually assumed to be Gaussian distributed ${ }^{1-3}$ when first-order perturbation theory of the soliton ${ }^{4-6}$ is used. Previous studies proved that the non-Gaussian timing jitter is induced by soliton interactions ${ }^{7-9}$ and regeneration ${ }^{10,11}$ but not by amplifier noise alone. When a first-order soliton perturbation is linearized, ${ }^{1,2}$ the GH timing jitter is indeed Gaussian distributed. However, if the equations from first-order perturbation are not linearized, as shown later, the amplitude, frequency, and timing jitters are all non-Gaussian distributed.

From the first-order perturbation theory of a soliton, ${ }^{2,4-6}$ with amplifier noise alone, the soliton parameters evolve according to the following stochastic differential equations (SDEs):

$$
\begin{aligned}
& \frac{\mathrm{d} A}{\mathrm{~d} \zeta}=n_{A}(\zeta), \\
& \frac{\mathrm{d} \Omega}{\mathrm{d} \zeta}=n_{\Omega}(\zeta), \\
& \frac{\mathrm{d} T}{\mathrm{~d} \zeta}=-\Omega+n_{T}(\zeta) .
\end{aligned}
$$

The phase perturbation is not shown in Eqs. (1)-(3) because it is not used here. Equations (1)-(3) are assumed Stratonovich but not as Ito SDEs. ${ }^{12}$ All the noise terms of $n_{A}(\zeta), n_{\Omega}(\zeta)$, and $n_{T}(\zeta)$ are independent Gaussian processes with autocorrelations of ${ }^{6,9}$

$$
\begin{aligned}
& E\left[n_{A}\left(\zeta_{1}\right) n_{A}\left(\zeta_{2}\right)\right]=A{\sigma_{n}}^{2} \delta\left(\zeta_{1}-\zeta_{2}\right), \\
& E\left[n_{\Omega}\left(\zeta_{1}\right) n_{\Omega}\left(\zeta_{2}\right)\right]=\frac{A}{3}{\sigma_{n}}^{2} \delta\left(\zeta_{1}-\zeta_{2}\right), \\
& E\left[n_{T}\left(\zeta_{1}\right) n_{T}\left(\zeta_{2}\right)\right]=\frac{\pi^{2}}{12 A^{3}}{\sigma_{n}}^{2} \delta\left(\zeta_{1}-\zeta_{2}\right),
\end{aligned}
$$

where $\sigma_{n}^{2}$ is the noise variance ${ }^{2}$ and $E[\cdot]$ denotes expectation. From Eqs. (4) to (6) the variances depend on the amplitude of $A(\zeta)$ and amplitude jitter transfers to both frequency and timing jitter. If the amplitude in the variances of Eqs. (4)-(6) is assumed to be constant $\left[A(\zeta) \approx A(0)=A_{0}\right]$ as a first-order approximation, amplitude, frequency, and timing jitters are indeed Gaussian distributed. The inclusion of amplitude jitter in Eqs. (4) -(6) is still within the assumption of the first-order soliton perturbation theory. ${ }^{4-6}$ The nonlinear first-order perturbation can be interpreted as repeated usage of the linearized first-order perturbation. ${ }^{2}$

Based on Eqs. (1) and (4) with an initial value of $A(0)=A_{0}$, the amplitude jitter is

$$
A(\zeta)=\left(A_{0}{ }^{1 / 2}+\frac{w_{A}}{2}\right)^{2}
$$

as a noncentral chi-square-distributed random process $^{3,11,13,14}$ with a variance parameter of $1 / 4 \sigma_{n}^{2} \zeta$, where $w_{A}$ is a Wiener process with an autocorrelation of $E\left[w_{A}\left(\zeta_{1}\right) w_{A}\left(\zeta_{2}\right)\right]=\sigma_{n}{ }^{2} \min \left(\zeta_{1}, \zeta_{2}\right)$.

Using Eqs. (2) and (5) yields a frequency jitter of

$$
\Omega(\zeta)=\int_{0}^{\zeta}\left(A_{0}^{1 / 2}+\frac{w_{A}\left(\zeta_{1}\right)}{2}\right) \mathrm{d} w_{\Omega},
$$

with a variance of

$$
\sigma_{\Omega}^{2}(\zeta)=\frac{1}{3} A_{0}{\sigma_{n}}^{2} \zeta+\frac{1}{24}{\sigma_{n}}^{4} \zeta^{2}
$$

where $w_{\Omega}$ is a Wiener process with an autocorrelation of $E\left[w_{\Omega}\left(\zeta_{1}\right) w_{\Omega}\left(\zeta_{2}\right)\right]=1 / 3 \sigma_{n}{ }^{2} \min \left(\zeta_{1}, \zeta_{2}\right)$ and is independent of the Wiener process of $w_{A}$.

The timing jitter in Eq. (3) has two terms. The first term of $-\Omega$ gives the $\mathrm{GH}$ timing jitter and increases with $\zeta^{3}$, and the second term of $n_{T}(\zeta)$ is just the projection of amplifier noise into the timing jitter. ${ }^{1}$ The first term of the GH timing jitter is far more interesting than the second. The SDE of $\mathrm{d} T_{\mathrm{GH}}=-\Omega \mathrm{d} \zeta$ has a solution of

$$
T_{\mathrm{GH}}(\zeta)=-\int_{0}^{\zeta}\left(\zeta-\zeta_{1}\right)\left[A_{0}{ }^{1 / 2}+\frac{w_{A}\left(\zeta_{1}\right)}{2}\right] \mathrm{d} w_{\Omega},
$$

with a variance of

$$
\sigma_{T}^{2}(\zeta)=\frac{1}{9} A_{0}{\sigma_{n}}^{2} \zeta^{3}+\frac{1}{144} \sigma_{n}^{4} \zeta^{4} .
$$

Similar to option pricing with stochastic volatility, ${ }^{15}$ the characteristic functions of frequency $\Omega(\zeta)$ and $\mathrm{GH}$ timing jitter $T_{\mathrm{GH}}(\zeta)$ are

$$
\Psi_{\Omega(\zeta)}(\nu)=G_{1}\left(\frac{\nu^{2}{\sigma_{n}}^{2}}{6}\right),
$$




$$
\begin{aligned}
\Psi_{T_{\mathrm{GH}}(\zeta)}(\nu)= & G_{2}\left(\frac{\nu^{2} \sigma_{n}{ }^{2}}{6}\right) \\
G_{1}(\lambda)= & E\left(\exp \left\{-\lambda \int_{0}^{\zeta}\left[A_{0}{ }^{1 / 2}+\frac{w_{A}\left(\zeta_{1}\right)}{2}\right]^{2} \mathrm{~d} \zeta_{1}\right\}\right) \\
G_{2}(\lambda)= & E\left(\operatorname { e x p } \left\{-\lambda \int_{0}^{\zeta}\left(\zeta-\zeta_{1}\right)^{2}\right.\right. \\
& \left.\left.\times\left[A_{0}{ }^{1 / 2}+\frac{w_{A}\left(\zeta_{1}\right)}{2}\right]^{2} \mathrm{~d} \zeta_{1}\right\}\right)
\end{aligned}
$$

where $G_{1}(-\lambda)$ and $G_{2}(-\lambda)$ are the moment generating functions of $\int_{0}^{\zeta}\left(A_{0}^{1 / 2}+1 / 2 w_{A}\right)^{2} \mathrm{~d} \zeta_{1}$ and $\int_{0}^{\zeta}\left(\zeta-\zeta_{1}\right)^{2}\left(A_{0}{ }^{1 / 2}+1 / 2 w_{A}\right)^{2} \mathrm{~d} \zeta_{1}$, respectively.

From the Cameron-Martin integral ${ }^{16}$ we obtain

$$
\begin{aligned}
& G_{1}(\lambda)=\frac{\exp \left[-\frac{2 A_{0}}{\sigma_{n}} \sqrt{\lambda} \tanh \left(\frac{\zeta \sigma_{n}}{2} \sqrt{\lambda}\right)\right]}{\cosh ^{1 / 2}\left(\frac{\zeta \sigma_{n}}{2} \sqrt{\lambda}\right)}, \\
& G_{2}(\lambda)=\left[\frac{f_{\lambda}(\zeta)}{f_{\lambda}(0)}\right]^{1 / 2} \exp \left(\lambda^{2}{\sigma_{n}}^{2} A_{0} \beta^{2}-\lambda A_{0} \zeta^{3} / 3\right),
\end{aligned}
$$

where

$$
\begin{aligned}
\beta^{2} & =\int_{0}^{\zeta}\left[\frac{1}{f_{\lambda}\left(\zeta_{1}\right)} \int_{\zeta_{1}}^{\zeta}\left(\zeta-\zeta_{2}\right)^{2} f_{\lambda}\left(\zeta_{2}\right) \mathrm{d} \zeta_{2}\right]^{2} \mathrm{~d} \zeta_{1}, \\
f_{\lambda}\left(\zeta_{1}\right) & =\sqrt{\zeta-\zeta_{1}} \mathrm{I}_{-1 / 4}\left[\frac{\sqrt{\lambda} \sigma_{n}}{2}\left(\zeta-\zeta_{1}\right)^{2}\right],
\end{aligned}
$$

where $I_{\nu}(\cdot)$ is the $\nu$ th-order modified Bessel function of the first kind. The function of $G_{1}(\lambda)$ [Eq. (16)] is similar to the characteristic function of nonlinear phase noise. $^{17,18}$ Using $\int_{0}^{1} x^{\nu+1} I_{\nu}(a x) \mathrm{d} x=I_{\nu+1}(a) / a,{ }^{19}$ we obtain

$$
\begin{aligned}
& \int_{\zeta_{1}}^{\zeta}\left(\zeta-\zeta_{2}\right)^{2} f_{\lambda}\left(\zeta_{2}\right) \mathrm{d} \zeta_{2}= \frac{\left(\zeta-\zeta_{1}\right)^{3 / 2}}{\sqrt{\lambda} \sigma_{n}} \\
& \times I_{3 / 4}\left[\frac{\sqrt{\lambda} \sigma_{n}}{2}\left(\zeta-\zeta_{1}\right)^{2}\right],(20) \\
& \beta^{2}=\sqrt{2}\left(\sqrt{\lambda} \sigma_{n}\right)^{-7 / 2} \int_{0}^{\sqrt{\lambda} \sigma_{n} \zeta^{2} / 2} x^{1 / 2} I_{3 / 4}{ }^{2}(x) I_{-1 / 4}{ }^{-2}(x) \mathrm{d} x .
\end{aligned}
$$

If the amplitude jitter is approximated as $A(\zeta) \approx A_{0}$ in both variances of Eqs. (4) and (5), we obtain

$$
\begin{aligned}
& G_{1}(\lambda) \approx \exp \left(-\lambda A_{0} \zeta\right), \\
& G_{2}(\lambda) \approx \exp \left(-\frac{1}{3} \lambda A_{0} \zeta^{3}\right),
\end{aligned}
$$

which are valid for high signal-to-noise ratio (SNR) of $\rho_{\mathrm{O}}=A_{0} /\left(\sigma_{n}^{2} \zeta\right)$. With approximations (22), the characteristic functions of Eqs. (12) and (13) are zero-mean Gaussian characteristic functions with variances of Eqs. (9) and (11), respectively, without the second term. The second term in Eqs. (9) and (11) increases the jitter variances by fractions $1 / 8 \rho_{\mathrm{O}}{ }^{-1}$ and $1 / 16 \rho_{\mathrm{O}}{ }^{-1}$, respectively.
From the frequency jitter in Eq. (8), the non-Gaussian distribution is induced by the term of $1 / 2 \int_{0}^{\zeta} w_{A} \mathrm{~d} w_{\Omega}$, i.e., the noise and noise interaction. The second-order soliton perturbation also includes noise and noise interaction. ${ }^{20,21}$ However, Eqs. (1)-(3) with noise variances from Eqs. (4) and (5) are directly from the first-order perturbation of a soliton. ${ }^{4-6}$ Similarly, the non-Gaussian timing jitter of Eq. (10) is induced by the term of $1 / 2 \int_{0}^{\zeta}\left(\zeta-\zeta_{1}\right) w_{A} \mathrm{~d} w_{\Omega}$, which also includes noise-noise interaction.

The probability density functions of frequency and GH timing jitter are the inverse Fourier transforms of the corresponding characteristic functions of Eqs. (12) and (13), respectively. Figure 1 plots the probability density of frequency and GH timing jitter compared with the Gaussian distribution with variances of Eqs. (9) and (11), respectively. The probability density functions are shown for SNRs of $\rho_{\mathrm{O}}=10$ and 20. The horizontal axis is normalized with respect to the standard deviation of frequency $\sigma_{\Omega}(\zeta)$ and timing $\sigma_{T}(\zeta)$ jitter [see Eqs. (9) and (11)] for Figs. 1(a) and $1(\mathrm{~b})$, respectively. Because the characteristic functions of Eqs. (12) and (13) are even real functions, the probability density functions are also even functions. Figure 1 plots only the positive frequency and timing jitters. Comparison of the frequency jitter of Fig. 1(a) and the timing jitter of Fig. 1(b) shows that
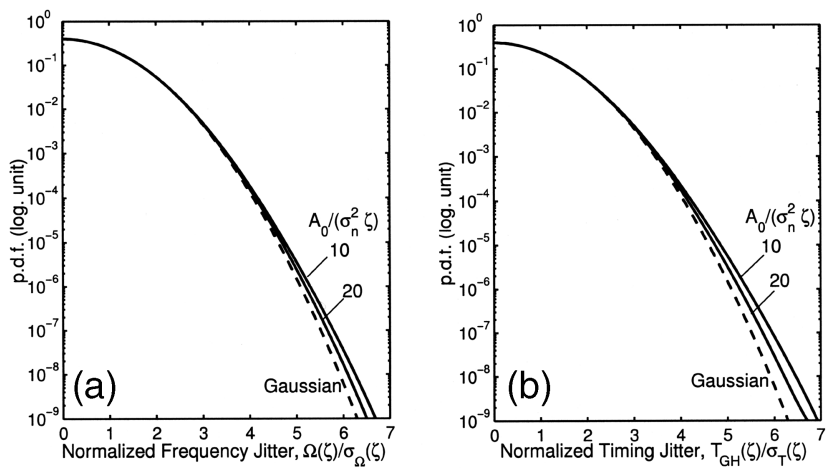

Fig. 1. Probability density functions of (a) frequency and (b) timing jitters compared with a Gaussian distribution (dashed curve).
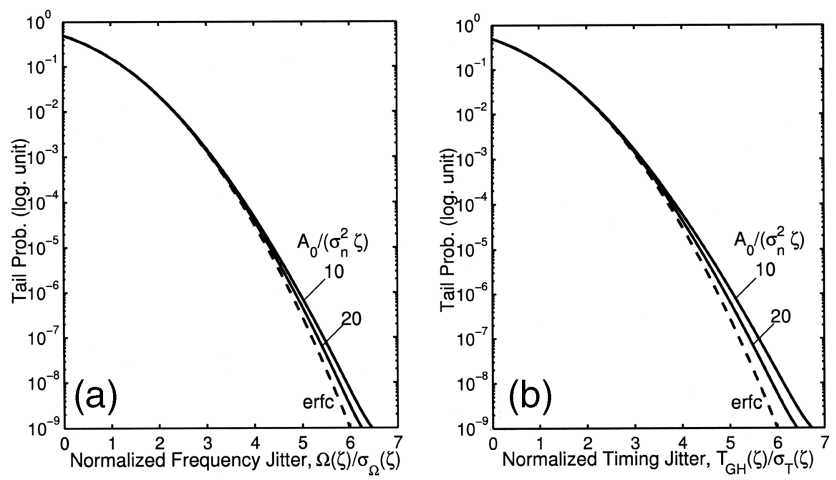

Fig. 2. Tail probabilities of (a) frequency and (b) timing jitters compared with a complementary error function (dashed curve). 
the frequency jitter has a distribution closer to the Gaussian distribution than does the timing jitter.

Figure 2 plots the tail probabilities that correspond to the probability density functions of Fig. 1 . The tail probability is defined as $\int_{x}^{\infty} p(x) \mathrm{d} x$ for a probability density function of $p(x)$. The tail probability is compared to the complementary error function of $1 / 2 \operatorname{erfc}(x / \sqrt{2})$, that is, the tail probability of the Gaussian distribution in Fig. 1. Comparing the tail probability of the frequency jitter of Fig. 2(a) and the timing jitter of Fig. 2(b), the frequency jitter is closer to the Gaussian distribution than is the timing jitter.

Both Figs. 1 and 2 are plotted on a logarithmic scale to show the difference at the tail distribution of both frequency and timing jitters to the Gaussian distribution. The non-Gaussian distribution leads to higher error probability than that of a Gaussian distribution. Figure 2(b) shows that the GH limit ${ }^{2}$ for an error probability of $10^{-9}$ decreases by $7 \%$ [from 6.00 to 6.42 of $\sigma_{T}(\zeta)$ ] and by $13 \%$ [from 6.00 to 6.76 of $\sigma_{T}(\zeta)$ ] for $\rho_{\mathrm{O}}=20$ and 10, respectively. As a comparison, the timing jitter standard deviation [see Eq. (11)] increases by factors of $0.16 \%$ and $0.31 \%$ for $\rho_{\mathrm{O}}=20$ and 10 , respectively.

Both Figs. 1(b) and 2(b) show that the distribution of GH timing jitter differs from the Gaussian distribution at the tail. Comparison of the generating function of Eq. (17) and the Gaussian approximation of (22) yields the non-Gaussian timing jitter distribution of $\left[f_{\lambda}(\zeta) / f_{\lambda}(0)\right]^{1 / 2} \exp \left(\lambda^{2} \sigma_{n}^{2} A_{0} \beta^{2}\right)$, which is obviously non-Gaussian.

In conclusion, based on the first-order soliton perturbation theory, both frequency and timing jitters were found to be non-Gaussian distributed. If the perturbation equations are linearized, amplitude, frequency, and timing jitters would all be Gaussian distributed. The timing jitter distribution differs more from a Gaussian distribution than does the frequency jitter.
K.-P. Ho's e-mail address is kpho@cc.ee.ntu.edu.tw.

\section{References}

1. J. P. Gordon and H. A. Haus, Opt. Lett. 11, 865 (1986).

2. E. Iannone, F. Matera, A. Mecozzi, and M. Settembre, Nonlinear Optical Communication Networks (Wiley, New York, 1998), Chap 5.

3. R. O. Moore, G. Biondini, and W. L. Kath, Opt. Lett. 28, 105 (2003).

4. Y. S. Kivshar and B. A. Malomed, Rev. Mod. Phys. 61, 763-915 (1989).

5. D. J. Kaup, Phys. Rev. A 42, 5689 (1990).

6. T. Georges, Opt. Fiber Technol. 1, 97 (1995).

7. C. R. Menyuk, Opt. Lett. 20, 285 (1995).

8. T. Georges, Electron. Lett. 31, 1174 (1995).

9. T. Georges, Opt. Commun. 123, 617 (1996).

10. O. Leclerc, E. Desurvire, P. Brindel, and E. Maunand, Opt. Fiber Technol. 5, 301 (1999).

11. G. E. Falkovich, I. Kolokolov, V. Lebedev, and S. K. Turisyn, Phys. Rev. E 63, 025601 (2001).

12. C. W. Gardiner, Handbook of Stochastic Methods, 2nd ed. (Springer-Verlag, Berlin, 1985).

13. P. A. Humblet and M. Azizog̃lu, J. Lightwave Technol. 9, 1576 (1991).

14. P. Shum and H. Ghafouri-Shiraz, Opt. Laser Technol. 28, 535 (1996).

15. E. M. Stein and J. C. Stein, Rev. Financ. Stud. 4, 727 (1991).

16. R. H. Cameron and W. T. Martin, Bull. Am. Math. Soc. 51, 73 (1945).

17. K.-P. Ho, in Advances in Optics and Laser Research, Vol. 3, W. T. Arkin, ed. (Nova Science, Hauppauge, N.Y., 2003).

18. K.-P. Ho, Opt. Lett. 28, 1350 (2003).

19. I. S. Gradshteyn and I. M. Ryzhik, Tables of Integrals, Series, and Products (Academic, San Diego, Calif., 1980), Sect. 6.561.

20. D. J. Kaup, Phys. Rev. A 44, 4582 (1991).

21. H. A. Haus, W. S. Wong, and F. I. Khatri, J. Opt. Soc. Am. B 14, 304 (1997). 